\title{
Impact of hematologic complete response in the treatment of sporadic late-onset nemaline myopathy associated with monoclonal gammopathy
}

\author{
Tania Maia ${ }^{1}$, Rui Bergantim ${ }^{1}$, Henrique Costa $^{2}$, Jorge Pinheiro ${ }^{1}$, and Fernanda Trigo ${ }^{1}$ \\ ${ }^{1}$ Centro Hospitalar de Sao Joao EPE \\ ${ }^{2}$ Centro Hospitalar e de Vila Nova de Gaia e Espinho
}

March 30, 2021

\begin{abstract}
Monoclonal gammopathy of undetermined significance (MGUS) may be associated with pathologies with severe neuromuscular manifestations such as sporadic late-onset nemaline myopathy (SLONM). We describe a difficult to diagnose case of SLNOM with marked clinical improvement after achieving gammopathy complete hematologic response.
\end{abstract}

\section{TITLE}

Impact of hematologic complete response in the treatment of sporadic late-onset nemaline myopathy associated with monoclonal gammopathy

\section{AUTHORS}

Tânia Maia ${ }^{1}$, Rui Bergantim ${ }^{1,2,3,4}$, Henrique Costa $^{5}$, Jorge Pinheiro ${ }^{6}$, Fernanda Trigo ${ }^{1}$

1. Hematology Department, Centro Hospitalar e Universitário São João, Porto, Portugal;

2. i3S - Instituto de Investigação e Inovação em Saúde, Universidade do Porto, Porto, Portugal;

3.Cancer Drug Resistance Group, IPATIMUP - Institute of Molecular Pathology and Immunology of the University of Porto, Porto, Portugal;

4. Hematology \& Oncology Unit, Department of Medicine - Faculty of Medicine of University of Porto, Porto, Portugal;

5. Neurology Department, Centro Hospitalar e de Vila Nova de Gaia e Espinho, Portugal.

6. Pathology Department, Centro Hospitalar e Universitário São João, Porto, Portugal.

Corresponding Author : Rui Bergantim (rui.bergantim@gmail.com)

Present address: Serviço de Hematologia Clínica - Centro Hospitalar São João; Alameda Prof. Hernâni Monteiro, 4200-319 Porto, Portugal

\section{KEY CLINICAL MESSAGE}

Monoclonal gammopathy of undetermined significance (MGUS) may be associated with pathologies with severe neuromuscular manifestations such as sporadic late-onset nemaline myopathy (SLONM). We describe a difficult to diagnose case of SLNOM with marked clinical improvement after achieving gammopathy complete hematologic response 


\section{KEYWORDS}

sporadic late-onset nemaline myopathy, monoclonal gammopathy of undetermined significance, autologous stem cell transplant

\section{INTRODUCTION}

Monoclonal gammopathy of undetermined significance (MGUS) may be associated with pathologies with severe neuromuscular manifestations, namely POEMS Syndrome and AL Amyloidosis[1-4]. Another lessknown association is with a rare disorder named sporadic late-onset nemaline myopathy (SLONM) [5-10] and happens in more than half of these patients[11, 12]. Diagnosis is confirmed by muscle biopsy with detection of intracytoplasmic nemaline rod bodies, representing Z-disc disorganization products that immunoreact with alfa-actinin and myotilin, accompanied by progressive atrophy of the muscle fiber[5, 11]. Necrotic or regenerating fibers and inflammatory infiltration are usually absent[13]. Clinically, SLONM appears mostly after the fourth decade of life as a rapidly progressing tetraparesis, mostly by proximal/axial muscle weakness with features such as dropped head syndrome, facial, bulbar involvement (dysphagia) and respiratory insufficiency leading to severe disability or death $[5,11,12]$. Head drop, neck muscle weakness, or neck muscle pain are features described in more than half of the patients with SLONM associated with MGUS (SLONMMGUS)[13]. Cardiac involvement is less common, but there are echocardiographic and electric conduction cardiac abnormalities in some patients $[3,11-16]$. The first cases published suggested an autoimmune etiology, and it was uncertain whether monoclonal gammopathy was associated or coincidental[6]. Treatment of first cases using immunosuppressive strategies showed better results with intravenous immunoglobulin[11, 17], mostly on non-MGUS associated cases[12]. Nonetheless, other immunosuppressive treatments (prednisone, cyclophosphamide, rituximab) or even plasmapheresis showed disappointing and inconstant outcomes which led the autoimmune etiology to be questioned, at least for some patients[3, 5, 6, 10, 14, 18, 19]. The hypothesis that SLONM-MGUS is a continuum of light-chain deposition disease (LCDD) is corroborated by the presence of light chain deposition in skeletal muscles observed in some cases described [20]. However, LCDD is a multiple organ disease while SLONM attainment is restricted to muscles, which could be an argument in favor of autoimmune abnormalities in its pathogenesis[13]. AL amyloidosis and POEMS are usually treated targeting the plasma cell clone with clinical improvement, as any manifestation associated with a plasma cell dyscrasia as postulated to SLONM-MGUS. [21-24]. The fast progression and severity of SLONM-MGUS (mortality of 70\% in 1-5 years) led clinicians to use the AL Amyloidosis treatment approach by using the autologous stem cell transplant (ASCT) with good results and low mortality rate $(2-3 \%)[5,12$, 25]. There are successful cases with clinical and histological improvement, as well as an increment in survival after conditioning with melphalan and ASCT, suggesting a possible toxic action of monoclonal protein as a degenerative mechanism for neurologic symptoms[14, 19, 20, 26-28]. Some propose light and heavy chains, rather than nemaline bodies, as responsible for myotoxic effects, and there is a report of coexistence of nemaline myopathy and amyloid myopathy[20, 29]. Furthermore, the evidence is growing about the association between disease severity and the size of the monoclonal protein supporting the rationale to use therapeutics targeting the clonal plasma cells[11, 12, 14, 28]. Other effective treatment options beyond ASCT, used in plasma cell dyscrasias can also be efficient, such as bortezomib[3, 14, 20, 30], lenalidomide[28, 31], cyclophosphamide or thalidomide[32]. Hematologic therapy seems to be the one that allows a lower risk of progression of the disease 24 months after treatment[15].

\section{CASE REPORT}

A 60-year-old female with no personal or family history of myopathy started in November/2014 to feel cervical pain in the shoulder girdle and upper limbs. Over three months, she developed muscle weakness resulting in proximal myopathy with drop-head and tetraparesis of proximal predominance. In July/2015, there was worsening of symptoms with dysphonia (ocular and tongue movements were regular) and unintentional weight loss (10 kg in 8 months). She also presented a myopathic gate with hyperlordosis, posterior pelvic tilt and bilateral winged scapula with hypotrophy of proximal muscles at the limbs without fasciculations (Figure 1A). Tendon reflexes and sensory perception were preserved. No respiratory involvement was evident, and her spirometry was normal. Human immunodeficiency virus and hepatitis serologies, autoimmune and active 
neoplastic screenings were negative. Normal levels of myoglobin, aldolase, creatine phosphokinase, lactate dehydrogenase and thyroid hormones were found. No abnormalities in echocardiogram or electrocardiogram were noted. Serum protein electrophoresis revealed an M-protein IgG lambda spike of $12 \mathrm{~g} / \mathrm{L}$ with normal immunoglobulin levels, normal free light chain ratio, negative urinary immunofixation and no bone marrow plasmacytosis. Anemia, hypercalcemia, renal dysfunction, and bone attainment were absent. Amyloid substance exploration was negative. Therefore, a monoclonal gammopathy of undetermined significance (MGUS) was diagnosed. Needle electromyography (EMG) showed myopathic pattern of the action potentials of the motor unity in all muscles explored, especially severe in cervical and the scapular girdle. However, there were no slow waves and fibrillation at rest. Left biceps brachii muscle biopsy demonstrated marked variation in fiber size with many atrophic angulated fibers containing numerous reddish granules representing positive rods in Gomori trichrome and immunohistochemistry for myotilin. The atrophic fibers were mainly of type 1; there was no necrosis, fibrosis or inflammatory infiltrate (Figure 2). The association of SLOMN and monoclonal gammopathy was assumed as a monoclonal gammopathy of neurological significance (MGNS) rather than an unknown significance.

In December/2015, collection of peripheral hematopoietic precursors after mobilization with granulocyte colony-stimulating factor (G-CSF) was performed obtaining 2.02x10^6 CD34+cells $/ \mathrm{kg}$. In January/2016, was submitted to ASCT after conditioning with melphalan $140 \mathrm{mg} / \mathrm{m} 2$. Neuromuscular deficits got worse in the acute phase after ASCT, with stabilization at eight months of follow-up. Due to the persistence of the monoclonal gammopathy at day 100 evaluation after ASCT, treatment with lenalidomide plus dexamethasone was started in September/2016. The patient recovered from general status, but after the fourth cycle, this trend was reversed occurring weight lost. Neuromuscular dysfunction with kyphotic posture remained stable until May/2017. By that time asthenia got worse accompanied by weight lost, myopathy aggravation with de novo hyperreflexia and respiratory insufficiency. With this evolution, lenalidomide was discontinued on the $9^{\text {th }}$ cycle. Noninvasive ventilation (NIV) and third-line treatment targeting the plasma cell clone with cyclophosphamide, bortezomib and dexamethasone (CyBorDex) were started in July/2017 for a total of eight cycles until January/2018. For the first time, after four cycles of CyBorDex, serum immunofixation got negative. Patient strength significantly improved, regaining the capacity of head elevation (Figure 1B), greater autonomy in daily life activities at home and a weight recovery of 12 kilograms over the CyBorDex treatment (Figure 3) again. The patient kept stable since the start of this treatment, reaching a weight of 54.7 kilograms and achieved the independence of nocturn NIV. The monoclonal gammopathy remains in complete remission three years after finishing treatments.

\section{DISCUSSION}

In the cases reported in the literature, there is a high rate of hematological and clinical responses to ASCT treatment with some patients responding only to the second ASCT[13, 28]. The clinical case we present initiated treatment 14 months after disease onset and did not achieve a good response after ASCT, showing some intensification of the myopathy after transplant followed by a stabilization and later slight improvement in autonomy in the daily life activities.

The most significant revision of transplanted SLONM-MGUS patients identified, as predictors of inadequate response to ASCT, the long course of the disease before the hematological treatment and the persistence of gammopathy after treatment[28], which were present in our clinical case. Furthermore, the increase of the M protein level precedes clinical deterioration, and there is a correlation between clinical course and amount of protein detected in peripheral blood[11,28]. Age at onset, light chain type of the monoclonal protein (kappa vs lambda) and severity of muscle weakness are not associated with a specific outcome[28].

Delayed ASCT may have affected response to treatment. However, according to these data which suggests a correlation between clinical course and gammopathy response, the goal of treatment should always be a complete response with suppression of the neoplastic clone, as soon as possible, to reduce the monoclonal protein and its toxic effects. Efficacy of Chemotherapy toward plasma cell dyscrasia without stem-cell transplantation has been reported as well[13]. In order to deepen hematological response to achieve a parallel clinical improvement, patients should receive additional Chemotherapy targeting plasma cell clone. Our clinical case 
illustrates the importance of implementing several treatments, such as proteasome inhibitors and immunomodulators, to obtain a complete hematologic response and consequently, the best clinical improvement. The complete hematologic response was achieved with the third line of treatment, allowing stabilization of muscle weakness and an impressive recovery of body weight. This approach supports the rationale of treating the monoclonal gammopathy to get the best neurological symptoms response [19, 20, 26, 28]. Whether or not nemaline rods diminished in our patient with treatment is not known, as a muscle biopsy was not performed after clinical improvement.

This clinical case shows that proteasome inhibitors and immunomodulators might be an effective therapy of SLONM related to monoclonal gammopathy in cases without a good response to ASCT or for patients with comorbidities who are not eligible for ASCT.

Even though the nature of the relationship between SLONM and monoclonal gammopathy is not entirely clarified, the correlation between clinical response and hematological response suggests a direct association between M protein and the disorder[13, 14]. All of these data have transformed SLONM associated with monoclonal gammopathy in a treatable disease with therapy directed toward plasma cell dyscrasia rather than immunosuppressive drugs used in autoimmune diseases[14]. The goal to eradicate clonal plasma cells, similar to other plasma cell dyscrasias makes logical the proposition to classify SLONM associated with monoclonal gammopathy as a monoclonal gammopathy with neurological significance (MGNS) and as a plasma cell dyscrasia with toxic monoclonal protein, similar to AL amyloidosis or POEMS [13, 28].

\section{CONSENT}

Verbal and written consent was obtained from the patient for use in this case for publication purposes. All data used in this case report was anonymized.

\section{CONFLICTS OF INTEREST}

RB: grants from Celgene, SPH/APCL/Amgen; advisory board and speaker honoraria from Celgene, Janssen, Takeda and Amgen. FT: grant SPH/APCL/Amgen; advisory board and speaker honoraria from Celgene, Janssen, Takeda and Amgen.

\section{AUTHOR'S CONTRIBUTIONS}

TM performed data collection and written the manuscript. RB was the patient primary hematologist, was involved in diagnosis, treatment plan and follow, and revised the manuscript. JP and HC were involved in the diagnosis, revised the data and the drafted paper. FT revised and supervised the drafted paper.

\section{REFERENCES}

1. Kuwabara, S., et al., Autologous peripheral blood stem cell transplantation for POEMS syndrome. Neurology, 2006. 66 (1): p. 105-107.

2. Ostrow, L.W., et al., Expanding the spectrum of monoclonal light chain deposition disease in muscle. Muscle \& Nerve, 2012.45 (5): p. 755-761.

3. Belhomme, N., et al., Rare myopathy associated to MGUS, causing heart failure and responding to chemotherapy. Annals of Hematology, 2017. 96 (4): p. 695-696.

4. Fermand, J.-P., et al., Monoclonal gammopathy of clinical significance: a novel concept with therapeutic implications. Blood, 2018. 132 (14): p. 1478-1485.

5. Chahin, N., D. Selcen, and A.G. Engel, Sporadic late onset nemaline myopathy. Neurology, 2005. 65 (8): p. 1158-1164.

6. Eymard, B., et al., Late-onset rod myopathy associated with monoclonal gammopathy. Neuromuscular Disorders, 1993. 3 (5-6): p. 557-560. 
7. Engel, A.G., Late-onset rod myopathy (a new syndrome?): light and electron microscopic observations in two cases. Mayo Clinic proceedings, 1966. 41 (11): p. 713-41.

8. Engel, W.K. and M.A. Oberc, Abundant Nuclear Rods in Adult-Onset Rod Disease. Journal of Neuropathology \& Experimental Neurology, 1975. 34 (2): p. 119-132.

9. Gyure, K.A., R.A. Prayson, and M.L. Estes, Adult-onset nemaline myopathy: a case report and review of the literature. Archives of pathology \& laboratory medicine, 1997. 121 (11): p. 1210-3.

10. Maeda, M.H., et al., Sporadic late-onset nemaline myopathy as a rare cause of slowly progressive muscle weakness with young adult onset. Muscle \& Nerve, 2015. 51 (5): p. 772-774.

11. Monforte, M., et al., Sporadic late-onset nemaline myopathy: clinical, pathology and imaging findings in a single center cohort.Journal of Neurology, 2018. 265 (3): p. 542-551.

12. Schnitzler, L.J., et al., Sporadic late-onset nemaline myopathy: clinico-pathological characteristics and review of 76 cases. Orphanet Journal of Rare Diseases, 2017. 12 (1): p. 86.

13. Uruha, A. and O. Benveniste, Sporadic late-onset nemaline myopathy with monoclonal gammopathy of undetermined significance.Current Opinion in Neurology, 2017. 30 (5): p. 457-463.

14. Kotchetkov, R., A. Dyszkiewicz-Korpanty, and V. Kukreti, Chemotherapy with stem cell transplantation is more effective than immunotherapy in sporadic late onset nemaline myopathy with monoclonal gammopathy. Bone Marrow Transplantation, 2018. 53 (7): p. 895-899.

15. Naddaf, E., et al., Sporadic late-onset nemaline myopathy: Clinical spectrum, survival, and treatment outcomes. Neurology, 2019.93 (3): p. e298-e305.

16. Broch, K., et al., A Middle-Aged Man Presenting With Progressive Heart Failure, Myopathy, and Monoclonal Gammopathy of Uncertain Significance. JACC: Case Reports, 2020. 2 (5): p. 785-789.

17. Milone, M., et al., Sporadic late onset nemaline myopathy responsive to IVIg and immunotherapy. Muscle \& Nerve, 2010.41 (2): p. 272-276.

18. Keller, C.E., et al., Adult-Onset Nemaline Myopathy and Monoclonal Gammopathy. Archives of Neurology, 2006. 63 (1): p. 132-134.

19. Voermans, N.C., et al., SPORADIC LATE-ONSET NEMALINE MYOPATHY EFFECTIVELY TREATED BY MELPHALAN AND STEM CELL TRANSPLANTSYMBOL.Neurology, 2008. 71 (7): p. $532-534$.

20. Doppler, K., et al., Sporadic late onset nemaline myopathy and immunoglobulin deposition disease. Muscle \& Nerve, 2013.48 (6): p. 983-988.

21. Dalakas, M.C. and S.A. Smith, A "nema" of hope in the treatment of late-onset nemaline myopathySYMBOL. Neurology, 2008.71 (7): p. 472-473.

22. Matsuzaki, K., et al., Marked improvement by high-dose chemotherapy and autologous stem cell transplantation in a case of light chain deposition disease. Journal of Nephrology, 2011. 24 (2): p. 246-249.

23. Weichman, K., et al., Clinical and molecular characteristics of patients with non-amyloid light chain deposition disorders, and outcome following treatment with high-dose melphalan and autologous stem cell transplantation. Bone Marrow Transplantation, 2006.38 (5): p. 339-343.

24. Merlini, G. and M.J. Stone, Dangerous small B-cell clones.Blood, 2006. 108 (8): p. 2520-30.

25. Jantunen, E., et al., Early treatment-related mortality in adult autologous stem cell transplant recipients: a nation-wide survey of 1482 transplanted patients. European Journal of Haematology, 2006.76 (3): p. 245-250. 
26. Benveniste, O., et al., STEM CELL TRANSPLANTATION IN A PATIENT WITH LATE-ONSET NEMALINE MYOPATHY AND GAMMOPATHYSYMBOLSYMBOL.Neurology, 2008. 71 (7): p. 531-532.

27. Novy, J., et al., Chemotherapy is successful in sporadic late onset nemaline myopathy (SLONM) with monoclonal gammopathy. Muscle \& Nerve, 2010. 41 (2): p. 286-287.

28. Voermans, N.C., et al., Sporadic late-onset nemaline myopathy with MGUS. Neurology, 2014. 83 (23): p. $2133-2139$.

29. Wang, M., et al., Monoclonal gammopathy with both nemaline myopathy and amyloid myopathy. Neuromuscular Disorders, 2017.27 (10): p. 942-946.

30. Schneider, R., et al., Clinical Reasoning: A 64-year-old man with progressive paraspinal muscle weakness. Neurology, 2016.86 (1): p. e4-e9.

31. Montagnese, F., et al., Sporadic late-onset nemaline myopathy in a woman with multiple myeloma successfully treated with lenalidomide/dexamethasone. Muscle \& Nerve, 2015. 51 (6): p. 934-935.

32. Kumutpongpanich, T., et al., Sporadic late-onset nemaline myopathy with monoclonal gammopathy of undetermined significance (SLONM-MGUS): An alternative treatment using cyclophosphamide-thalidomidedexamethasone (CTD) regimen. Neuromuscular Disorders, 2018. 28 (7): p. 610-613.

\section{FIGURES LEGENDS}

Figure 1: A ) Dropped head and proximal dominant limb weakness with kyphotic posture sustained before autologous stem cell transplant. B ) Notable amelioration of cervical muscular force, not adopting a kyphotic posture, after cyclophosphamide, bortezomib and dexamethasone.

Figure 2: Muscle biopsy with marked variation in fiber size and clusters of intracytoplasmic rods, often filling atrophic fibers, with no inflammatory cells, are the pathological hallmark. A : Gomori 100x; B : Gomori 400x; C : Myotilin Immunohistochemistry 200x; D : Electron dense rod in electron microscopy

Figure 3 . Bodyweight and muscle strength variation during clinical evolution and treatments.

(ASCT: autologous transplant; CyBorDex: cyclophosphamide, bortezomib, dexamethasone ; DTR: deep tendon reflexes ; Flex/ext: cervical flexion/extension; Hyper: hyperreflexia; IMNF: immunofixation in peripheral blood; Kg: kilograms; Len/dex: lenalidomide and dexamethasone; LL: lower limb; N: normal; Prox: proximal; Resp ins: respiratory insufficiency; Rot R / L: cervical rotation to the right/to the left ; UL: upper limb) Strength measured according to MRC ( Medical Research Council) scale; blue line: body weigh variation; orange shaded area: immunofixation negativity; green line: treatments) 
Figure 1
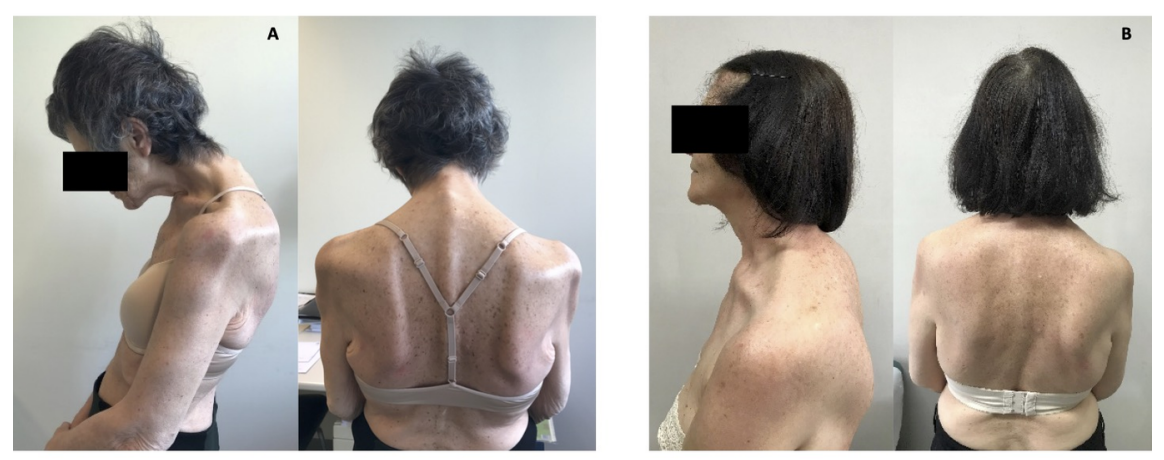

Figure 2
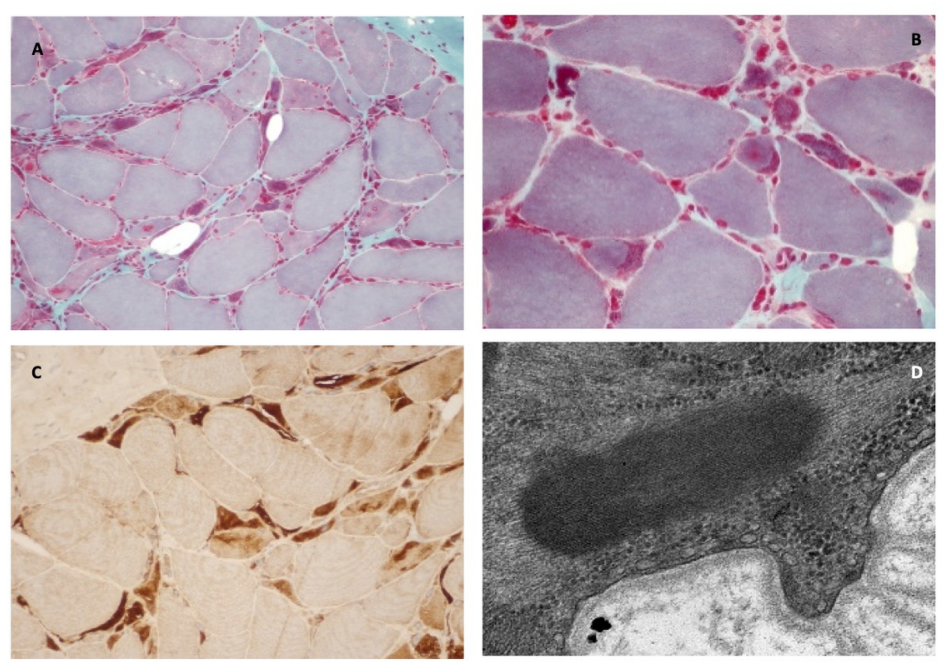
Figure 3

\begin{tabular}{|l|llllllll|}
\hline Weight (Kg) \\
\hline Muscle
\end{tabular}

